\title{
Ramucirumab plus FOLFIRI or irinotecan as second-line therapy in advanced or metastatic gastric or gastroesophageal junction adenocarcinoma
}

\author{
Verena Schlintl, Florian Huemer, Richard Greil, Lukas Weiss \\ Department of Internal Medicine III with Haematology, Medical Oncology, Haemostaseology, Infectiology, and Rheumatology, Oncologic Center, \\ Salzburg Cancer Research Institute, Laboratory for Immunological and Molecular Cancer Research (SCRI-LIMCR), Paracelsus Medical University \\ Salzburg, Salzburg, Austria \\ Correspondence to: Lukas Weiss, MD, PhD. Department of Internal Medicine III with Haematology, Medical Oncology, Haemostaseology, \\ Infectiology, and Rheumatology, Oncologic Center, Salzburg Cancer Research Institute, Laboratory for Immunological and Molecular Cancer \\ Research (SCRI-LIMCR), Paracelsus Medical University Salzburg, Salzburg, Austria. Email: lu.weiss@salk.at. \\ Response to: Vogl UM, Vormittag L, Winkler T, et al. Ramucirumab plus paclitaxel or FOLFIRI in platinum-refractory advanced or metastatic gastric \\ or gastroesophageal junction adenocarcinoma-experience at two centres. J Gastrointest Oncol 2020;11:366-75.
}

Submitted Jun 05, 2020. Accepted for publication Oct 22, 2020.

doi: 10.21037 /jgo-20-230

View this article at: http://dx.doi.org/10.21037/jgo-20-230

We read with great interest the article by Vogl et al. published in the Fournal of Gastrointestinal Oncology on a twocentre experience of ramucirumab (RAM) plus paclitaxel or FOLFIRI in advanced gastric/gastroesophageal junction (GEJ) cancer (1). The authors could show a favourable outcome for RAM plus FOLFIRI in a selected cohort $(n=16)$ of taxane-refractory patients or patients with pre-existing neurotoxicity. We can corroborate these findings with a retrospective analysis of 16 patients treated with RAM plus irinotecan or FOLFIRI at our tertiary cancer centre.

The addition of the anti-vascular endothelial growth factor receptor 2 antibody RAM to paclitaxel has been shown to prolong survival in advanced or metastatic gastric/GEJ cancer with disease progression on or after fluoropyrimidine- or platinum-containing chemotherapy (2). The results of the RAINBOW trial led to the approval of RAM in this indication by the Food and Drug Administration as well as by the European Medicines Agency and RAM plus paclitaxel nowadays constitutes the standard second-line therapy. Since the conduction of this pivotal trial, the treatment landscape of localized gastric/GEJ cancer has changed considerably. The FLOT regimen (fluorouracil, oxaliplatin, docetaxel) has replaced EOX (epirubicin, oxaliplatin, capecitabin) as standard perioperative chemotherapy for operable localized gastric/ GEJ cancer (3). This regimen has also been adopted by many centres as first-line therapy in patients with advanced/ metastatic disease and high tumour volume and/or symptomatic disease (4).

However, approximately $70 \%$ of patients undergoing FLOT therapy will suffer from peripheral neuropathy (PNP), which impedes further application of potentially neurotoxic agents in clinical practice (3). In addition, progression under or rapid disease recurrence after FLOT in the perioperative or first-line setting raises the demand for an alternative chemotherapy backbone in subsequent treatment other than paclitaxel (5). We-as others-have therefore combined RAM with FOLFIRI or with irinotecan in analogy to the RAISE trial in colorectal cancer (6).

\section{Data analysis and findings}

This is a retrospective analysis of 16 patients with advanced or metastatic gastric/GEJ cancer, who received off-label treatment with RAM plus FOLFIRI or irinotecan at our institution between January 2015 and April 2020. Patients aged 18 years or older with histologically confirmed gastric or GEJ adenocarcinoma were considered eligible. RAM was administered at $8 \mathrm{mg} / \mathrm{kg}$ body weight, irinotecan at $180 \mathrm{mg} / \mathrm{m}^{2}$ and FOLFIRI in combination with 5 -fluoruracil (5-FU) $400 \mathrm{mg} / \mathrm{m}^{2}$ bolus and $2,400 \mathrm{mg} / \mathrm{m}^{2}$ over 46 hours continuously, biweekly. Palliative treatment was classified as 
Table 1 Baseline characteristics of patients treated with ramucirumab plus FOLFIRI or irinotecan at treatment initiation

\begin{tabular}{|c|c|c|c|}
\hline \multirow{3}{*}{ Characteristic } & \multicolumn{3}{|c|}{ No. of patients [\%] } \\
\hline & Total & $\begin{array}{c}\text { RAM + } \\
\text { FOLFIRI }\end{array}$ & $\mathrm{RAM}+\mathrm{IRI}$ \\
\hline & 16 & $9[56]$ & $7[44]$ \\
\hline Median age y [range] & $55[46-71]$ & $54[51-68]$ & $58[51-71]$ \\
\hline \multicolumn{4}{|l|}{ Sex } \\
\hline Male & 12 [75] & $7[88]$ & $5[71]$ \\
\hline Female & $4[25]$ & $2[22]$ & $2[29]$ \\
\hline \multicolumn{4}{|l|}{ ECOG PS } \\
\hline 0 & $2[13]$ & $2[22]$ & $0[0]$ \\
\hline 1 & 10 [63] & $4[44]$ & $6[86]$ \\
\hline 2 & $4[25]$ & $3[33]$ & $1[14]$ \\
\hline \multicolumn{4}{|l|}{ Primary site of disease } \\
\hline Gastric & $8[50]$ & $3[33]$ & $5[71]$ \\
\hline Gastroesophageal junction & $8[50]$ & $6[67]$ & $2[29]$ \\
\hline \multicolumn{4}{|l|}{ Resection of primary tumor } \\
\hline Yes & 11 [69] & 8 [89] & $3[43]$ \\
\hline \multicolumn{4}{|l|}{ Perioperative chemotherapy } \\
\hline Yes & 12 [75] & 8 [89] & $4[57]$ \\
\hline \multicolumn{4}{|l|}{ Prior palliative therapy lines } \\
\hline 0 & 10 [63] & 7 [78] & $3[43]$ \\
\hline 1 & $5[31]$ & 2 [22] & $3[43]$ \\
\hline$\geq 2$ & $1[6]$ & $0[0]$ & $1[14]$ \\
\hline \multicolumn{4}{|c|}{ Subsequent palliative therapy lines } \\
\hline 0 & $11[69]$ & $6[67]$ & $5[71]$ \\
\hline$\geq 1$ & $5[31]$ & $3[33]$ & $2[29]$ \\
\hline \multicolumn{4}{|c|}{ Time point of metastases detection } \\
\hline Synchronous & $5[31]$ & $2[22]$ & $3[43]$ \\
\hline Metachronous & $11[69]$ & $7[78]$ & $4[57]$ \\
\hline \multicolumn{4}{|l|}{ Peritoneal metastases } \\
\hline Yes & $6[38]$ & $3[33]$ & $3[43]$ \\
\hline \multicolumn{4}{|l|}{ Reason for treatment choice } \\
\hline Pre-existing PNP & $5[31]$ & $1[11]$ & $4[57]$ \\
\hline Early progression & 10 [63] & $7[78]$ & $3[43]$ \\
\hline Other & $1[6]$ & $1[11]$ & $0[0]$ \\
\hline
\end{tabular}

RAM, ramucirumab; IRI, irinotecan; PS, performance score; PNP, peripheral neuropathy. first-line therapy in regard to evidence of metastatic disease, irrespective of interval from perioperative chemotherapy for localized disease. Progression-free survival (PFS) was calculated from the date of therapy start until radiologically confirmed progression or death. Patients without progression at the last contact were censored. Overall survival (OS) was calculated from the date of therapy start until death from any cause. Patients alive at the last contact were censored. Overall response rate (ORR) was evaluated using Response Evaluation Criteria in Solid Tumours (RECIST 1.1) (7). Adverse events were evaluated according to the Common Terminology Criteria for Adverse Events (CTCAE version 4.0). Baseline characteristics were analysed descriptively. The median PFS and OS were determined using the Kaplan-Meier method. Statistical analyses were performed using SPSS for Windows v23 (IBM, Armonk, NY, USA). P values $<0.05$ were considered to indicate statistical significance.

In total, 16 patients with advanced or metastatic gastric/ GEJ cancer were treated with RAM plus FOLFIRI or irinotecan at our institution. Patient baseline characteristics are summarized in Table 1.

Median age was 55 years ranging between 46 and 71 years. One-quarter of patients was 65 years or older. Threequarters of patients were male. Eastern Cooperative Oncology Group (ECOG) performance status (PS) at start of RAM-based therapy was $\geq 1$ in the majority of our cohort $(88 \%)$. Primary site of disease was equally distributed to stomach and gastroesophageal junction. Most of primary tumours received perioperative systemic therapy $(75 \%)$ and had undergone oncologic resection (69\%). Peritoneal metastases were present in $38 \%$ of patients $(n=6)$. All patients had received prior 5 -FU based therapy. Subsequent palliative therapy was applied in $31 \%$ of patients $(n=5)$ and consisted of checkpoint-inhibitors, trifluridine/tipiracil or cytotoxic monotherapy. FOLFIRI-RAM or irinotecanRAM was chosen due to progression during or rapid disease recurrence after FLOT in the perioperative or first-line setting in $63 \%(\mathrm{n}=10)$ and pre-existing PNP CTCAE grade $>1$ in $31 \%(\mathrm{n}=5)$.

The median PFS and OS of all patients were 5.4 (95\% CI: 3.7-7.1) and 7.6 (95\% CI: 6.1-9.1) months, respectively. The clinical benefit rate was $63 \%(n=10)$ with an ORR of $38 \%(n=6)$. Only one patient discontinued RAMbased therapy because of treatment toxicity. Presence of peritoneal metastases at treatment initiation was not associated with a statistically significant impact on survival rates. Patients with rapid recurrence, which was defined as 


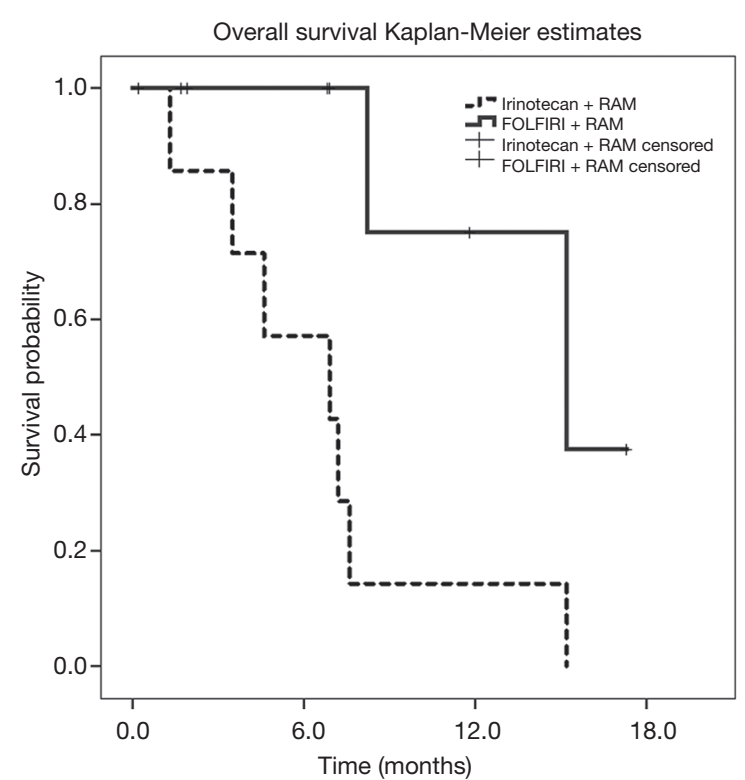

Figure 1 Kaplan-Meier curves for OS according to chemotherapy backbone in combination with RAM in 16 patients with advanced gastric/GEJ cancer. FOLFIRI plus RAM: $n=9$, irinotecan plus RAM: n=7; OS, overall survival; GEJ, gastroesophageal junction.

disease progression within six months after perioperative chemotherapy, did not show a statistically significant worse outcome in comparison to the rest of our cohort (median OS: 7.6 vs. 7.2 months, $\mathrm{P}=0.82$ ). Median time to relapse after perioperative chemotherapy in patients with initially curative setting was 3.8 months.

Patients receiving FOLFIRI-RAM displayed a statistically significantly longer OS compared to irinotecanRAM [median: 15.2 months (95\% CI: 4.7-25.7) vs. 6.9 months (95\% CI: $1.0-12.8), \mathrm{P}=0.01$, Figure 1]. There was no statistically significant difference in PFS (median: 5.4 vs. 4.6 months, $\mathrm{P}=0.19$ ). Age ( $t$-test $\mathrm{P}=0.44)$, sex (chi-square $\mathrm{P}=0.77$ ), ECOG PS (chi-square $\mathrm{P}=0.51$ ), number of prior (chi-square $\mathrm{P}=0.27$ ) and subsequent (chi-square $\mathrm{P}=0.41$ ) palliative therapy lines were balanced between FOLFIRIRAM and irinotecan-RAM treated subgroups.

\section{Critical discussion and future perspectives}

Our results are consistent with the findings of Vogl et al. and support RAM plus FOLFIRI or irinotecan as treatment option for advanced gastric/GEJ cancer in patients with pre-existing PNP, progression during or rapid recurrence following FLOT. A median OS of 7.6 months in this real-world cohort was comparable to the results from the RAINBOW trial (2). Cytotoxic monotherapy with irinotecan in second-line treatment of advanced/metastatic gastric/GEJ cancer is known to improve median OS up to 4.0 months in comparison to best-supportive care (7). The significantly prolonged survival with FOLFIRI as chemotherapy backbone compared to irinotecan in our single-centre cohort might suggest a clinical benefit of 5-FU continuation in combination with RAM and irinotecan. However, results have to be interpreted with caution due to the limited number of patients $(n=16)$. We did not detect statistically significant imbalances of prognostic baseline characteristics between the two chemotherapy backbone groups. However, the majority of patients receiving irinotecan-RAM had been pre-treated with palliative intent, whereas FOLFIRI-RAM was mostly administered as first-line therapy. Due to the retrospective character of this analysis, comprehensive evaluation of adverse events according to CTCAE was not feasible. Half of the patients who had progressed during RAM-based therapy $(n=5)$ underwent at least one further palliative treatment line, which underlines tolerability as shown by the RAISE trial in patients with colorectal cancer (6).

The multicenter randomized phase 2 RAMIRIS trial has recently proven feasibility and PFS benefit of FOLFIRI plus RAM in taxane-pre-treated patients with advanced gastric/GEJ cancer (8). Results of the phase 3 study (NCT03081143) should clarify the clinical efficacy in this setting and are eagerly awaited.

\section{Acknowledgments}

Funding: None.

\section{Footnote}

Provenance and Peer Review: This article was a free submission to the journal. The article has undergone external peer review.

Peer Review File: Available at http://dx.doi.org/10.21037/ jgo-20-230

Conflicts of Interest: All authors have completed the ICMJE uniform disclosure form (available at http://dx.doi. org/10.21037/jgo-20-230). FH and LW report personal fees from Lilly, outside the submitted work. The other authors have no conflicts of interest to declare. 
Ethical Statement: The authors are accountable for all aspects of the work in ensuring that questions related to the accuracy or integrity of any part of the work are appropriately investigated and resolved. This analysis was approved by the Ethics Committee of the provincial government of Salzburg (415-EALL/5/39-2019).

Open Access Statement: This is an Open Access article distributed in accordance with the Creative Commons Attribution-NonCommercial-NoDerivs 4.0 International License (CC BY-NC-ND 4.0), which permits the noncommercial replication and distribution of the article with the strict proviso that no changes or edits are made and the original work is properly cited (including links to both the formal publication through the relevant DOI and the license). See: https://creativecommons.org/licenses/by-nc-nd/4.0/.

\section{References}

1. Vogl UM, Vormittag L, Winkler T, et al. Ramucirumab plus paclitaxel or FOLFIRI in platinum-refractory advanced or metastatic gastric or gastroesophageal junction adenocarcinoma-experience at two centres. J Gastrointest Oncol 2020;11:366-75.

2. Wilke H, Muro K, Van Cutsem E, et al. Ramucirumab plus paclitaxel versus placebo plus paclitaxel in patients with previously treated advanced gastric or gastro-oesophageal junction adenocarcinoma (RAINBOW): a double-blind, randomised phase 3 trial. Lancet Oncol 2014;15:1224-35.

3. Al-Batran SE, Homann N, Pauligk C, et al. Perioperative chemotherapy with fluorouracil plus leucovorin, oxaliplatin, and docetaxel versus fluorouracil or capecitabine plus cisplatin and epirubicin for locally

Cite this article as: Schlintl V, Huemer F, Greil R, Weiss L. Ramucirumab plus FOLFIRI or irinotecan as second-line therapy in advanced or metastatic gastric or gastroesophageal junction adenocarcinoma. J Gastrointest Oncol 2021;12(2):906909. doi: 10.21037/jgo-20-230 advanced, resectable gastric or gastro-oesophageal junction adenocarcinoma (FLOT4): a randomised, phase 2/3 trial. Lancet 2019;393:1948-57.

4. Al-Batran SE, Hartmann JT, Hofheinz R, et al. Biweekly fluorouracil, leucovorin, oxaliplatin, and docetaxel (FLOT) for patients with metastatic adenocarcinoma of the stomach or esophagogastric junction: a phase II trial of the Arbeitsgemeinschaft Internistische Onkologie. Ann Oncol 2008;19:1882-7.

5. Al-Batran SE, Homann N, Pauligk C, et al. Effect of Neoadjuvant Chemotherapy Followed by Surgical Resection on Survival in Patients With Limited Metastatic Gastric or Gastroesophageal Junction Cancer: The AIOFLOT3 Trial. JAMA Oncol 2017;3:1237-44.

6. Tabernero J, Yoshino T, Cohn AL, et al. Ramucirumab versus placebo in combination with second-line FOLFIRI in patients with metastatic colorectal carcinoma that progressed during or after first-line therapy with bevacizumab, oxaliplatin, and a fluoropyrimidine (RAISE): a randomised, double-blind, multicentre, phase 3 study. Lancet Oncol 2015;16:499-508.

7. Thuss-Patience PC, Kretzschmar A, Bichev D, et al. Survival advantage for irinotecan versus best supportive care as second-line chemotherapy in gastric cancer--a randomised phase III study of the Arbeitsgemeinschaft Internistische Onkologie (AIO). Eur J Cancer 2011;47:2306-14.

8. Lorenzen S, Thuss-Patience PC, Pauligk C, et al. FOLFIRI plus ramucirumab versus paclitaxel plus ramucirumab as second-line therapy for patients with advanced or metastatic gastroesophageal adenocarcinoma with or without prior docetaxel: Results from the phase II RAMIRIS Study of the AIO. J Clin Oncol 2020;38:4514. 